\title{
Elastic Stability of Circular Arches with the Open Thin-walled Monosym- metric Section Considering the Prebuckling Deformation
}

\author{
Hyo-Jin Ryu ${ }^{1}$, Jin-Yu Choi ${ }^{2}$, Gyu-Sei Yi ${ }^{3}$, Chin-Ok Lee ${ }^{1}$, Nam-Hyoung Lim ${ }^{1, *}$ \\ ${ }^{1}$ Department of Civil Engineering, Chung Nam National University, 99 Daehak-ro, Yuseong-gu, Daejeon 305-764, \\ South Korea \\ ${ }^{2} R \& D$ Strategy Division, Korea Railroad Research Institute, 176 Cheoldo bangmulgwan-ro, Uiwang, Gyeonggi-do,437- \\ 757, South Korea \\ ${ }^{3}$ Department of Civil Engineering, Sun Moon University, 221 Sunmoon-ro, Tangjeong-Myeon, Asan-si, Chungnam 336- \\ 708, South Korea
}

\begin{abstract}
The effect of prebuckling in-plane deformations on the elastic flexural-torsional buckling of arches is studied in this paper. Nonlinear strain-displacement relations considering initial curvature effects and higher order prebuckling deformation terms with curvature in deriving process are substituted into the second variation of the total potential to obtain the buckling energy equation. The analytical solutions for the flexural-torsional buckling moment of arches in uniform bending, containing the effects of the prebuckling deformation, are proposed. Also, the influence of the higher order prebuckling deformation terms with the curvature effects is investigated according to the ratio of the minor axis flexural stiffness to the major axis flexural stiffness.
\end{abstract}

Keywords: Arch, Curvature effect, Elastic buckling, Flexural-torsional buckling, Prebuckling deformation, Thin-walled.

\section{INTRODUCTION}

In the classical analysis for the elastic flexural-torsional buckling of beams, it is assumed that the prebuckling inplane deformations are small enough to be neglected on the buckling load. This assumption is suitable only when the ratio of the minor axis flexural stiffness to the major axis flexural stiffness is very small. When the ratio of the minor axis flexural stiffness to the major axis flexural stiffness is not small, prebuckling in-plane deformations are known to increase the elastic buckling resistance of straight beams, because prebuckling deformations transform the straight beam into an arch. Previous theoretical investigations of the effects of the prebuckling in-plane deformations on the buckling moments of straight member have been studied by a number of researchers; Trahair and Woolcock [1], Roberts and Azizian [2], Pi and Trahair [3,4] and others.

The classical solution for the elastic buckling load of thin-walled circular arches has been studied by a number of researchers; Vlasov [5], Yoo [6], Papangelis and Trahair [7], Kang and Yoo [8], Pi and Bradford [9, 10], Lim and Kang [11], Bradford and Pi [12], Yang et al. [13] and others. The influence of prebuckling in-plane deformations on the elastic flexural-torsional buckling of arches in uniform bending was

\footnotetext{
*Address correspondence to this author at the Department of Civil Engineering, Chung Nam National University, 99 Daehak-ro, Yuseong-gu, Daejeon 305-764, South Korea; Tel: +82-42-821-7005; Fax: +82-42-825-0318; E-mail: nhrim@cnu.ac.kr
}

studied by Vacharajittiphan and Trahair [14], Yoo and Pfeiffer [15], and Pi et al. [16], and it was found that the prebuckling deformations increase the elastic flexural-torsional buckling resistance of simply supported arches. Pi and Bradford [17] presented closed form solutions for the elastic flexural-torsional buckling resistance of laterally fixed arches with a doubly symmetric section and investigated the effects of the prebuckling in-plane deformations. Pi and Bradford [18] investigated the prebuckling response of the classical analysis and its effects on determining the out-of-plane and in-plane elastic buckling loads of circular arches with a doubly symmetric section subjected to the uniform radial load. Some of the discrepancies between these researches are believed to be due to inappropriate interpretations of fundamental assumptions and to use of different degree of approximation of the curvature effects and prebuckling deformation effects in the derivation.

In this paper, buckling energy equation including the effect of the initial curvature and prebuckling deformation is proposed for monosymmteric circular arches. The analytical solutions for the flexural-torsional buckling load of laterally simply supported circular arches in uniform bending, containing the effects of the prebuckling deformation, are obtained and the results are compared with the previous theoretical solutions. Also, the influence of the higher order prebuckling deformation terms with the curvature effects is investigated according to the ratio of the minor axis flexural stiffness to the major axis flexural stiffness. 


\section{STRAINS AND DISPLACEMENTS}

The basic assumptions made in this study are as follows:

The cross sections retain their original shape.

(ii) The shear strains due to change of normal stresses, such as bending and warping normal stresses, are negligibly small.

(iii) The length of the beam is much larger than any other dimensions of the cross section.

(iv) The shear strains along the middle surface of the thin-walled cross section are negligibly small.

(v) The normal strain due to bending is linearly distributed in a cross section.

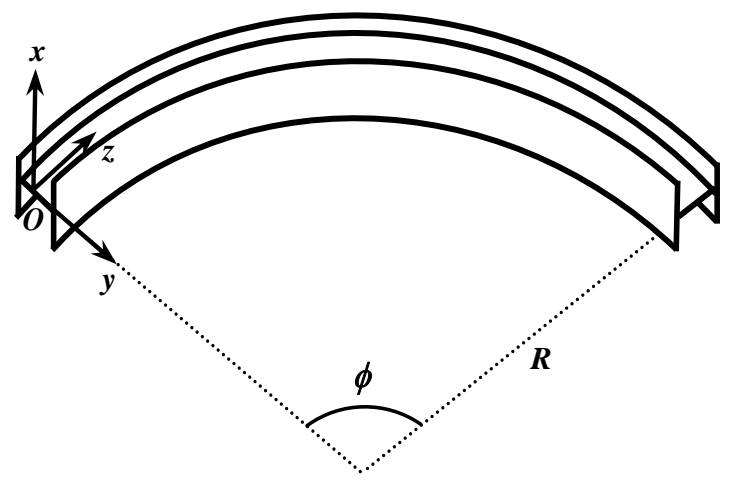

Fig. (1). Curvilinear coordinate system of arches.

Fig. (1) shows the curvilinear coordinate system of a circular arch that has an initial curvature in the direction of the minor principal axis oy of the cross-section. The longitudinal normal strain $\varepsilon$ in the system $(x, y, z)$ shown in Fig. (1) can be written as follows (Usami and Koh [19]; Kang and Yoo [8]; Lim and Kang [11]):

$\varepsilon=\left(\frac{R}{R-y}\right)\left(\frac{\partial w}{\partial z}-\frac{v}{R}\right)+\frac{1}{2}\left(\frac{R}{R-y}\right)^{2}\left[\left(\frac{\partial v}{\partial z}+\frac{w}{R}\right)^{2}+\left(\frac{\partial w}{\partial z}-\frac{v}{R}\right)^{2}+\left(\frac{\partial u}{\partial z}\right)^{2}\right]$

where $u, v$, and $w$ are the components of the displacement in the $x, y$, and $z$ directions, respectively. Note that the normal strain $\quad \varepsilon$ varies nonlinearly with $y$ as a result of the $(R / R-y)$ term. For the case of a curved beam subjected to bending, the normal strain distribution is shown in Fig. (2).

This strain variation is considerably different from that of a straight beam. The strain is definitely nonlinear; in fact, it is hyperbolic in the $y$ direction. This is due to the initial curvature of the beam. In the curved beam formula proposed by Oden [4], the nonlinearity of the strain is due to the quantity $(R / R-y)$. For example, machine parts, hooks, chain links, and gears may have $h / R$ ratios of near unity or larger, and their normal strain distribution must be evaluated with the nonlinearity. However, if the value of $h / R$ is small in comparison with unity, the strain distribution is essentially a linear function of $y$ (Oden [20]).

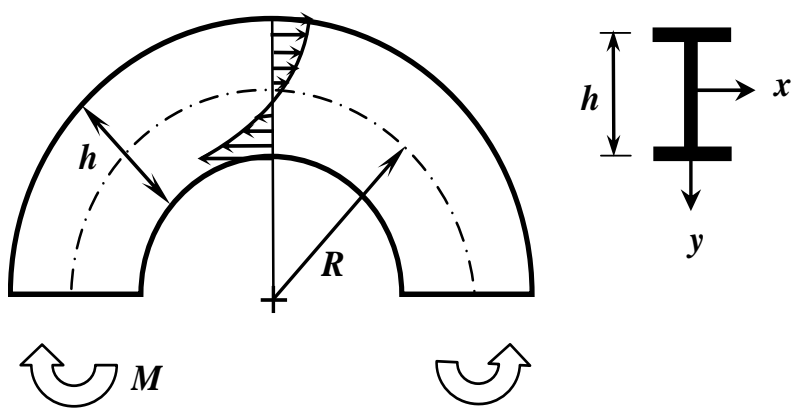

Fig. (2). Normal strain distribution in a cross section of curved beam.

Utilizing the result by Oden [20] and the real structural condition of an arch where the value of $h / R$ is small, the variation of $\varepsilon$ with $y$ is assumed to be linear with ignoring the $(R / R-y)$ term. With this assumption, the normal strain in Eq. (1) can be approximated as

$$
\varepsilon=\frac{\partial w}{\partial z}-\frac{v}{R}+\frac{1}{2}\left[\left(\frac{\partial v}{\partial z}+\frac{w}{R}\right)^{2}+\left(\frac{\partial w}{\partial z}-\frac{v}{R}\right)^{2}+\left(\frac{\partial u}{\partial z}\right)^{2}\right]
$$

The shear strain due to bending and warping of the thinwalled section are neglected. The shear strain due to uniform torsion is approximated by

$$
\gamma=2 n k
$$

where $n$ is the distance from the mid-thickness surface and $k$ is the twist.

The displacement components are functions of the coordinates, $\mathrm{x}, \mathrm{y}$, and $\mathrm{z}$. Using the approaches of Usami and Koh [19], the displacement at any point can be written in terms of shear center as

$$
\begin{aligned}
& u=u_{o}-\left(y-y_{o}\right) \theta-\frac{1}{2} x \theta^{2} \\
& v=v_{o}+x \theta-\left(y-y_{o}\right) \frac{1}{2} \theta^{2} \\
& w=w_{c}-x\left[\left(v_{o}^{\prime}+\frac{w_{c}}{R}\right) \theta+u_{o}^{\prime}-\frac{1}{2} u_{o}^{\prime} \theta^{2}\right]-y\left[\left(v_{o}^{\prime}+\frac{w_{c}}{R}\right)-u_{o}^{\prime} \theta-\frac{1}{2} \theta^{2}\left(v_{o}^{\prime}+\frac{w_{c}}{R}\right)\right] \\
&-\omega\left[\theta^{\prime}-\frac{u_{o}^{\prime}}{R}-\frac{\theta}{R}\left(v_{o}^{\prime}+\frac{w_{c}}{R}\right)+\frac{1}{2 R} u_{o}^{\prime} \theta^{2}\right]
\end{aligned}
$$

where $u_{o}$ and $v_{o}$ are a displacement of the shear center in the principal centroidal coordinate system (x,y); $\theta$ is a rotation of a cross section about the z-axis; $w_{c}$ is the longitudinal displacement of a cross section, which is the same for all points on a cross section, and is referred to as the average longitudinal displacement; $y_{o}$ is coordinate of the shear center; $\omega$ is the normalized warping function according to the principal sectorial coordinate system.

Substituting the displacements given in Eqs. (4a) (4c) into Eqs. (2) and (3), the nonlinear longitudinal normal strain 


$$
\begin{aligned}
\varepsilon= & \bar{w}_{c}^{\prime}-x \bar{u}_{o}^{\prime \prime}-y \bar{v}_{o}^{\prime \prime}-\omega \bar{\theta}^{\prime \prime}+\frac{1}{2}\left(u_{o}^{\prime 2}+\bar{v}_{o}^{\prime 2}+\bar{w}_{c}^{\prime 2}\right)+\frac{1}{2}\left[x^{2}+\left(y-y_{o}\right)^{2}\right] \theta^{\prime 2} \\
& +y_{o}\left(u_{o}^{\prime} \theta^{\prime}-\frac{1}{2 R} \theta^{2}+\bar{v}_{o}^{\prime} \theta \theta^{\prime}\right)+y\left(u_{o}^{\prime \prime} \theta-\frac{1}{R} \bar{v}_{o}^{\prime 2}-\bar{v}_{o}^{\prime \prime} \bar{w}_{c}^{\prime}+\frac{1}{2 R} \theta^{2}+\frac{1}{2} \bar{v}_{o}^{\prime \prime} \theta^{2}+\frac{1}{R} \bar{v}_{o}^{\prime} u_{o}^{\prime} \theta\right) \\
& -x\left(\bar{v}_{o}^{\prime \prime} \theta+\frac{1}{R} u_{o}^{\prime} \bar{v}_{o}^{\prime}+\bar{w}_{c}^{\prime} u_{o}^{\prime \prime}+\frac{1}{R} \bar{w}_{c}^{\prime} \theta\right)-y y_{o}\left(\frac{1}{R} \bar{v}_{o}^{\prime} \theta \theta^{\prime}-\frac{1}{2 R} \bar{v}_{o}^{\prime \prime} \theta^{2}\right)+\frac{\omega}{R}\left(\bar{v}_{o}^{\prime \prime} \theta+\bar{v}_{o}^{\prime} \theta^{\prime}-\bar{v}_{o}^{\prime} \bar{\theta}^{\prime}+\frac{1}{R} \bar{v}_{o}^{\prime 2} \theta\right) \\
& -x^{2}\left(\frac{1}{R} u_{o}^{\prime} \theta^{\prime}-\frac{1}{2 R^{2}} u_{o}^{\prime 2}-\frac{1}{2} u_{o}^{\prime 2}-\frac{1}{2} \theta^{\prime 2}-\frac{1}{R} u_{o}^{\prime \prime} \theta-\frac{1}{2 R^{2}} \theta^{2}-\frac{1}{R^{2}} \bar{v}_{o}^{\prime} u_{o}^{\prime} \theta-\frac{1}{R} \bar{v}_{o}^{\prime \prime} \theta^{2}-\bar{v}_{o}^{\prime} u_{o}^{\prime \prime} \theta^{\prime}-\bar{v}_{o}^{\prime \prime} u_{o}^{\prime \prime} \theta\right) \\
& -x \omega\left(\frac{1}{R} \bar{\theta}^{\prime 2}-\bar{\theta}^{\prime \prime} \bar{u}_{o}^{\prime \prime}+\frac{1}{R^{3}} \bar{v}_{o}^{\prime} u_{o}^{\prime} \theta-\frac{1}{R^{2}} \bar{v}_{o}^{\prime} \theta \bar{\theta}^{\prime}-\bar{v}_{o}^{\prime \prime} \theta \bar{\theta}^{\prime \prime}+\frac{1}{R} \bar{v}_{o}^{\prime \prime} u_{o}^{\prime \prime} \theta+\frac{1}{R^{2}} \bar{v}_{o}^{\prime \prime} \theta^{2}+\frac{1}{R} \bar{v}_{o}^{\prime} u_{o}^{\prime \prime} \theta^{\prime}-\bar{v}_{o}^{\prime} \theta^{\prime} \bar{\theta}^{\prime \prime}\right) \\
& -x y\left(\frac{1}{R} \bar{v}_{o}^{\prime} \bar{\theta}^{\prime}+\bar{v}_{o}^{\prime \prime} \bar{u}_{o}^{\prime \prime}-\frac{1}{R^{2}} \bar{v}_{o}^{\prime 2} \theta-\bar{v}_{o}^{\prime \prime 2} \theta-\bar{v}_{o}^{\prime} \bar{v}_{o}^{\prime \prime} \theta^{\prime}\right)+\frac{1}{2} y^{2}\left(\frac{1}{R} \bar{v}_{o}^{\prime 2}+\bar{v}_{o}^{\prime \prime 2}-2 \bar{v}_{o}^{\prime \prime} u_{o}^{\prime \prime} \theta-2 \bar{v}_{o}^{\prime \prime} u_{o}^{\prime} \theta^{\prime}+\frac{2}{R} \bar{v}_{o}^{\prime} \theta \theta^{\prime}-\frac{1}{R} \bar{v}_{o}^{\prime \prime} \theta^{2}\right) \\
& +y \omega\left(\frac{1}{R^{2}} \bar{v}_{o}^{\prime} \bar{\theta}^{\prime}+\bar{v}_{o}^{\prime \prime} \bar{\theta}^{\prime \prime}-\frac{1}{R^{3}} \bar{v}_{o}^{\prime 2} \theta-\frac{1}{R} \bar{v}_{o}^{\prime \prime 2} \theta-\frac{1}{R} \bar{v}_{o}^{\prime} \bar{v}_{o}^{\prime \prime} \theta^{\prime}\right)+\frac{1}{2} \omega^{2}\left(\frac{1}{R^{2}} \bar{\theta}^{\prime 2}+\bar{\theta}^{\prime \prime 2}-\frac{2}{R^{3}} \bar{v}_{o}^{\prime} \theta \bar{\theta}^{\prime}-\frac{2}{R} \bar{v}_{o}^{\prime \prime} \theta \bar{\theta}^{\prime \prime}-\frac{2}{R} \bar{v}_{o}^{\prime} \theta^{\prime} \bar{\theta}^{\prime \prime}\right)
\end{aligned}
$$

and shear strain at a point on the cross section are obtained as follows:

$$
\gamma=2 n\left(\bar{\theta}^{\prime}-\frac{1}{R} \theta \bar{v}_{o}^{\prime}+\frac{1}{2 R} u_{o}^{\prime} \theta^{2}\right)
$$

where

$$
\begin{array}{lr}
\bar{w}_{c}^{\prime}=w_{c}^{\prime}-\frac{v_{o}}{R}, \quad \bar{v}_{o}^{\prime}=v_{o}^{\prime}+\frac{w_{c}}{R}, \quad \bar{\theta}^{\prime}=\theta^{\prime}-\frac{u_{o}^{\prime}}{R}, \quad \bar{u}_{o}^{\prime \prime}=u_{o}^{\prime \prime}+\frac{\theta}{R}, \\
\bar{v}_{o}^{\prime \prime}=v_{o}^{\prime \prime}+\frac{w_{c}^{\prime}}{R}, \bar{\theta}^{\prime \prime}=\theta^{\prime \prime}-\frac{u_{o}^{\prime \prime}}{R} & (7 \mathrm{a}, \mathrm{b}, \mathrm{c}, \mathrm{d}, \mathrm{e}, \mathrm{f})
\end{array}
$$

\section{BUCKLING ENERGY EQUATIONS}

The following assumptions are adopted in this paper to obtain energy equation on the flexural-torsional buckling of arches considering prebuckling deformations.

(a) The last buckled positions on the flexural-torsional buckling consist of prebucked displacements and buckled displacements as follow;

$$
u_{o}=u_{o p}+u_{o b} ; v_{o}=v_{o p}+v_{o b} ; w_{c}=w_{c p}+w_{c b} ; \theta=\theta_{p}+\theta_{b}
$$

in which, " $b$ " and " $p$ " denote the bucking behavior and prebuckling behavior, respectively.

(b) Prior to flexural-torsional buckling, there are no out-ofplane displacements.

(c) Prior to flexural-torsional buckling, prebuckling strains are very small.

(d) In-plane displacements and stress resultants are constant during the flexural-torsional buckling.

(e) The axis of the arch is inextensible during the flexuraltorsional buckling.

\subsection{Prebuckling Stresses and Stress Resultants}

Prior to buckling, the displacements from assumption (a) and (b) are given by $u_{o}=0 ; \theta=0 ; v_{o}=v_{o p} ; w_{c}=w_{c p}$.
Also, from assumption (c), prebuckling strains due to prebuckling displacements can be used as linear function. Applying these conditions into strain-displacement relations of Eq. (5), prebuckling strains $\left(\varepsilon_{p}, \gamma_{p}\right)$, stresses $\left(\sigma_{p}, \tau_{p}\right)$ and stress resultants $\left(F_{z}, M_{x}\right)$ are then approximated by

$$
\begin{aligned}
& \varepsilon_{p}=\bar{w}_{c p}^{\prime}-y \bar{v}_{o p}^{\prime \prime}, \gamma_{p}=0 \\
& \sigma_{p}=E \varepsilon_{p}, \tau_{p}=0 \\
& F_{z}=\int_{A} \sigma_{p} d A=E A \bar{w}_{c p}^{\prime} \\
& M_{X}=\int_{A} y \sigma_{p} d A=-E I_{x} \bar{v}_{o p}^{\prime \prime}
\end{aligned}
$$

where $\bar{w}_{c p}^{\prime}=w_{c p}^{\prime}-v_{o p} / R, \quad \bar{v}_{o p}^{\prime \prime}=v_{o p}^{\prime \prime}+w_{c p}^{\prime} / R, A$ is the area of the cross section and $I_{X}$ is the moment of inertia about the major axis $o x$.

\subsection{Variations of strains}

As a result of assumptions (a), (b), and (d), buckling behavior of arches can be defined from the prebuckled position $\left(u_{o p}=0, v_{o p}, w_{c p}, \theta_{p}=0\right)$ to the buckled posi$\operatorname{tion}\left(u_{o b}, v_{o b}=0, w_{c b}=0, \theta_{b}\right)$. Also, the variation of prebuckling displacements is equal to zero from assumption (d), and $\bar{w}_{c p}^{\prime}=w_{c p}^{\prime}-v_{o p} / R=0$ due to inextensible condition of assumption (e).

By considering the above conditions, the first and second variations of strains are obtained as

$$
\begin{aligned}
\delta \varepsilon= & -x\left(\delta \bar{u}_{o b}^{\prime \prime}+\bar{v}_{o p}^{\prime \prime} \delta \theta_{b}+\frac{1}{R} \bar{v}_{o p}^{\prime} \delta u_{o b}^{\prime}\right)-\omega\left(\delta \bar{\theta}_{b}^{\prime \prime}-\frac{1}{R} \bar{v}_{o p}^{\prime \prime} \delta \theta_{b}-\frac{1}{R^{2}} \bar{v}_{o p}^{\prime} \delta u_{o b}^{\prime}\right) \\
& -x y\left(\frac{1}{R} \bar{v}_{o p}^{\prime} \delta \bar{\theta}_{b}^{\prime}+\bar{v}_{o p}^{\prime \prime} \delta \bar{u}_{o b}^{\prime \prime}\right)+y \omega\left(\frac{1}{R^{2}} \bar{v}_{o p}^{\prime} \delta \bar{\theta}_{b}^{\prime}+\bar{v}_{o p}^{\prime \prime} \delta \bar{\theta}_{b}^{\prime \prime}\right) \\
\delta \gamma & =2 n\left(\delta \theta_{b}^{\prime}-\frac{1}{R} \delta u_{o b}^{\prime}-\frac{1}{R} \bar{v}_{o p}^{\prime} \delta \theta_{b}\right)
\end{aligned}
$$




$$
\begin{aligned}
\delta^{2} \varepsilon= & \delta u_{o b}^{\prime 2}+\left[x^{2}+\left(y-y_{o}\right)^{2}\right] \delta \theta_{b}^{\prime 2}-y_{o}\left(\frac{1}{R} \delta \theta_{b}^{2}-2 \delta u_{o b}^{\prime} \delta \theta_{b}^{\prime}-2 \bar{v}_{o p}^{\prime \prime} \delta \theta_{b} \delta \theta_{b}^{\prime}\right) \\
& +y\left(2 \delta u_{o b}^{\prime \prime} \delta \theta_{b}+\frac{1}{R} \delta \theta_{b}^{2}+\bar{v}_{o p}^{\prime \prime} \delta \theta_{b}^{2}+\frac{2}{R} \bar{v}_{o p}^{\prime} \delta \theta_{b} \delta u_{o b}^{\prime}\right)-y y_{o}\left(\frac{2}{R} \bar{v}_{o p}^{\prime} \delta \theta_{b} \delta \theta_{b}^{\prime}-\frac{1}{R} \bar{v}_{o p}^{\prime \prime} \delta \theta_{b}^{2}\right) \\
& -x^{2}\left(\frac{2}{R} \delta u_{o b}^{\prime} \delta \theta_{b}^{\prime}-\frac{1}{R^{2}} \delta u_{o b}^{\prime 2}-\delta u_{o b}^{\prime \prime 2}-\delta{\theta_{b}^{\prime}}^{2}-\frac{2}{R} \delta \theta_{b} \delta u_{o b}^{\prime \prime}-\frac{1}{R^{2}} \delta \theta_{b}^{2}-\frac{2}{R^{2}} \bar{v}_{o p}^{\prime} \delta u_{o b}^{\prime} \delta \theta_{b}\right. \\
& \left.-2 \bar{v}_{o p}^{\prime \prime} \delta u_{o b}^{\prime \prime} \delta \theta_{b}-\frac{2}{R} \bar{v}_{o p}^{\prime \prime} \delta \theta_{b}^{2}-2 \bar{v}_{o p}^{\prime} \delta u_{o b}^{\prime \prime} \delta \theta_{b}^{\prime}\right)-2 x \omega\left(\frac{1}{R} \delta \bar{\theta}_{b}^{\prime 2}-\delta \bar{\theta}_{b}^{\prime \prime} \delta \bar{u}_{o b}^{\prime \prime}+\frac{1}{R^{3}} \bar{v}_{o p}^{\prime} \delta u_{o b}^{\prime} \delta \theta_{b}\right. \\
& \left.-\frac{1}{R^{2}} \bar{v}_{o p}^{\prime} \delta \theta_{b} \delta \bar{\theta}_{b}^{\prime}-\bar{v}_{o p}^{\prime \prime} \delta \theta_{b} \delta \bar{\theta}_{b}^{\prime \prime}-\bar{v}_{o p}^{\prime} \delta \theta_{b}^{\prime} \delta \bar{\theta}_{b}^{\prime \prime}+\frac{1}{R} \bar{v}_{o p}^{\prime \prime} \delta u_{o b}^{\prime \prime} \delta \theta_{b}+\frac{1}{R^{2}} \bar{v}_{o p}^{\prime \prime} \delta \theta_{b}^{2}+\frac{1}{R} \bar{v}_{o p}^{\prime} \delta u_{o b}^{\prime \prime} \delta \theta_{b}^{\prime}\right) \\
& -y^{2}\left(2 \bar{v}_{o p}^{\prime \prime} \delta u_{o b}^{\prime \prime} \delta \theta_{b}+2 \bar{v}_{o p}^{\prime \prime} \delta u_{o b}^{\prime} \delta \theta_{b}^{\prime}-\frac{2}{R} \bar{v}_{o p}^{\prime} \delta \theta_{b} \delta \theta_{b}^{\prime}+\frac{1}{R} \bar{v}_{o p}^{\prime \prime} \delta \theta_{b}^{2}\right) \\
& +\omega^{2}\left(\frac{1}{R^{2}} \delta \bar{\theta}_{b}^{\prime 2}+\delta \bar{\theta}_{b}^{\prime \prime 2}-\frac{2}{R^{3}} \bar{v}_{o p}^{\prime} \delta \theta_{b} \delta \bar{\theta}_{b}^{\prime}-\frac{2}{R} \bar{v}_{o p}^{\prime \prime} \delta \theta_{b} \delta \bar{\theta}_{b}^{\prime \prime}-\frac{2}{R} \bar{v}_{o p}^{\prime} \delta \theta_{b}^{\prime} \delta \bar{\theta}_{b}^{\prime \prime}\right)
\end{aligned}
$$

$\delta^{2} \gamma=0$

in which the higher order terms of prebuckling curvature and rotation containing $\bar{v}_{o p}^{\prime 2}, \bar{v}_{o p}^{\prime \prime 2}, \bar{v}_{o p}^{\prime} \bar{v}_{o p}^{\prime \prime}$ are neglected.

\subsection{Energy Equation}

Under the external moment $M_{e x}$, the principle of stationary potential energy can be represented by the following equation;

$$
\begin{aligned}
& \delta \Pi=\int_{V} \sigma \delta \varepsilon d V+\int_{V} \tau \delta \gamma d V-\sum_{0, L} M_{e x} \delta v^{\prime}=0 \quad(16) \quad \begin{array}{c}
\text { Substituting Eqs. (12) } \\
\text { the energy equation then }
\end{array} \\
& \delta^{2} \Pi=\int_{L}\left\{\sum_{y}\left(\delta \bar{u}_{o b}^{\prime \prime}+\bar{v}_{o p}^{\prime \prime} \delta \theta_{b}+\frac{1}{R} \bar{v}_{o p}^{\prime} \delta u_{o b}^{\prime}\right)^{2}+E I_{\omega}\left(\delta \bar{\theta}_{b}^{\prime \prime}-\frac{1}{R} \bar{v}_{o p}^{\prime \prime} \delta \theta_{b}-\frac{1}{R^{2}} \bar{v}_{o p}^{\prime} \delta u_{o b}^{\prime}\right)^{2}+W \delta \theta_{b}^{\prime 2}\right. \\
&+ G K_{T}\left(\delta \theta_{b}^{\prime}-\frac{1}{R} \delta u_{o b}^{\prime}-\frac{1}{R} \bar{v}_{o p}^{\prime} \delta \theta_{b}\right)^{2}+F_{z}\left(\delta u_{o b}^{\prime 2}+2 y_{o} \delta u_{o b}^{\prime} \delta \theta_{b}^{\prime}-\frac{1}{R} y_{o} \delta \theta_{b}^{2}+2 y_{o} \bar{v}_{o p}^{\prime} \delta \theta_{b} \delta \theta_{b}^{\prime}\right) \\
&+M_{x}\left(2 \delta u_{o b}^{\prime \prime} \delta \theta_{b}+\frac{1}{R} \delta \theta_{b}^{2}+\frac{2}{R} \bar{v}_{o p}^{\prime} \delta \theta_{b} \delta u_{o b}^{\prime}+\bar{v}_{o p}^{\prime \prime} \delta \theta_{b}^{2}-\frac{2}{R} y_{o} \bar{v}_{o p}^{\prime} \delta \theta_{b} \delta \theta_{b}^{\prime}+\frac{1}{R} y_{o} \bar{v}_{o p}^{\prime \prime} \delta \theta_{b}^{2}\right) \\
&+E J_{x x y y}\left(\frac{1}{R} \bar{v}_{o p}^{\prime} \delta \bar{\theta}_{b}^{\prime}+\bar{v}_{o p}^{\prime \prime} \delta \bar{u}_{o b}^{\prime \prime}\right)^{2}-2 E J_{x y y \omega}\left(\frac{1}{R} \bar{v}_{o p}^{\prime} \delta \bar{\theta}_{b}^{\prime}+\bar{v}_{o p}^{\prime \prime} \delta \bar{u}_{o b}^{\prime \prime}\right)\left(\frac{1}{R^{2}} \bar{v}_{o p}^{\prime} \delta \bar{\theta}_{b}^{\prime}+\bar{v}_{o p}^{\prime \prime} \delta \bar{\theta}_{b}^{\prime \prime}\right) \\
&+E J_{y y \omega \omega}\left(\frac{1}{R^{2}} \bar{v}_{o p}^{\prime} \delta \bar{\theta}_{b}^{\prime}+\bar{v}_{o p}^{\prime \prime} \delta \bar{\theta}_{b}^{\prime \prime}\right)^{2}+2 E J_{x x y}\left(\delta \bar{u}_{o b}^{\prime \prime}+\bar{v}_{o p}^{\prime \prime} \delta \theta_{b}+\frac{1}{R} \bar{v}_{o p}^{\prime} \delta u_{o b}^{\prime}\right)\left(\frac{1}{R} \bar{v}_{o p}^{\prime} \delta \bar{\theta}_{b}^{\prime}+\bar{v}_{o p}^{\prime \prime} \delta \bar{u}_{o b}^{\prime \prime}\right) \\
&-2 E J_{\omega \omega y}\left(\delta \bar{\theta}_{b}^{\prime \prime}-\frac{1}{R} \bar{v}_{o p}^{\prime \prime} \delta \theta_{b}-\frac{1}{R^{2}} \bar{v}_{o p}^{\prime} \delta u_{o b}^{\prime}\right)\left(\frac{1}{R^{2}} \bar{v}_{o p}^{\prime} \delta \bar{\theta}_{b}^{\prime}+\bar{v}_{o p}^{\prime \prime} \delta \bar{\theta}_{b}^{\prime \prime}\right) \\
&+K_{\omega}\left(\frac{2}{R} \delta u_{o b}^{\prime} \delta \theta_{b}^{\prime}-\frac{1}{R^{2}} \delta u_{o b}^{\prime 2}-\delta u_{o b}^{\prime \prime 2}-\delta \theta_{b}^{\prime 2}-\frac{2}{R} \delta \theta_{b} \delta u_{o b}^{\prime \prime}-\frac{1}{R^{2}} \delta \theta_{b}^{2}-\frac{2}{R^{2}} \bar{v}_{o p}^{\prime} \delta u_{o b}^{\prime} \delta \theta_{b}\right. \\
&\left.-2 \bar{v}_{o p}^{\prime \prime} \delta u_{o b}^{\prime \prime} \delta \theta_{b}-\frac{2}{R} \bar{v}_{o p}^{\prime \prime} \delta \theta_{b}^{2}-2 \bar{v}_{o p}^{\prime \prime} \delta u_{o b}^{\prime \prime} \delta \theta_{b}^{\prime}\right)-K_{x \omega}\left(\frac{2}{R} \delta \bar{\theta}_{b}^{\prime 2}-2 \delta \bar{\theta}_{b}^{\prime \prime} \delta \bar{u}_{o b}^{\prime \prime}+\frac{2}{R^{3}} \bar{v}_{o p}^{\prime} \delta u_{o b}^{\prime} \delta \theta_{b}\right. \\
&\left.\left.-\frac{2}{R^{2}} \bar{v}_{o p}^{\prime} \delta \theta_{b} \delta \bar{\theta}_{b}^{\prime}-2 \bar{v}_{o p}^{\prime \prime} \delta \theta_{b} \delta \bar{\theta}_{b}^{\prime \prime}-2 \bar{v}_{o p}^{\prime} \delta \theta_{b}^{\prime} \delta \bar{\theta}_{b}^{\prime \prime}+\frac{2}{R} \bar{v}_{o p}^{\prime \prime} \delta u_{o b}^{\prime \prime} \delta \theta_{b}+\frac{2}{R} \bar{v}_{o p}^{\prime \prime} \delta \theta_{b} \delta \bar{\theta}_{b}^{\prime \prime}-\frac{2}{R} \bar{v}_{o p}^{\prime} \delta \theta_{b}^{2}+\frac{2}{R} \bar{v}_{o p}^{\prime} \delta \bar{\theta}_{o b}^{\prime \prime} \delta \theta_{b}^{\prime}\right)\right\} \\
&\left.-K_{o b}^{\prime \prime} \delta \theta_{b}+2 \bar{v}_{o p}^{\prime \prime} \delta u_{o b}^{\prime} \delta \theta_{b}^{\prime}-\frac{2}{R} \bar{v}_{o p}^{\prime} \delta \theta_{b} \delta \theta_{b}^{\prime}+\frac{1}{R} \bar{v}_{o p}^{\prime \prime} \delta \theta_{b}^{2}\right)
\end{aligned}
$$

where $\delta$ represents the first variation; $V$ and $L$ denote the volume of the arch and the developed length of the arch, respectively; $\sigma$ and $\tau$ denote the longitudinal normal stress and shear stress, respectively. The critical state of equilibrium is that the second variation of the total potential energy, $\delta^{2} \Pi$ is equal to zero, which indicates a possible transition from a stable state to an unstable state. This energetic criterion of the buckling state can be written as

$$
\delta^{2} \Pi=\int_{V} \sigma \delta^{2} \varepsilon d V+\int_{V} \tau \delta^{2} \gamma d V+\int_{V} E(\delta \varepsilon)^{2} d V+\int_{V} G(\delta \gamma)^{2} d V=0
$$

Substituting Eqs. (12), (13), (14) and (15) into Eq. (17), the energy equation then becomes 


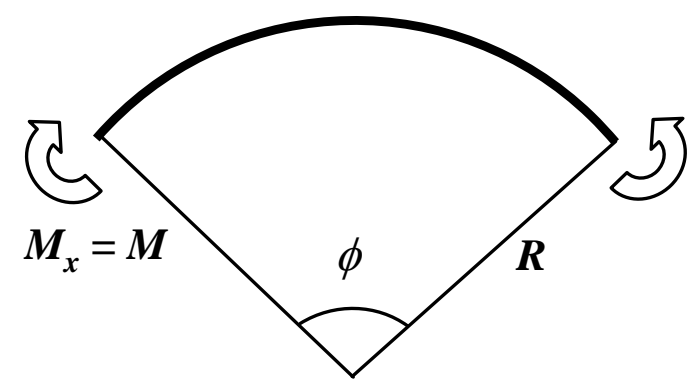

(a) Positive Bending

Fig. (3). Arches in uniform bending.

in which $F_{z}$ is the axial force; $M_{x}$ is the moment about $x$ axis; $I_{y}$ is the second moment of area about the minor axis oy; $I_{\omega}$ is the warping constant of the cross section; $G$ is the shear modulus of elasticity; $K_{T}$ is the Saint-Venant torsional

The stress resultants given in Eq. (18) is defined as

$$
\begin{aligned}
& K_{y}=\int_{A} \sigma x^{2} d A, \quad K_{x \omega}=\int_{A} \sigma x \omega d A, \quad K_{x}=\int_{A} \sigma y^{2} d A, \\
& K_{\omega}=\int_{A} \sigma \omega^{2} d A \\
& J_{x x y y}=\int_{A} x^{2} y^{2} d A, \\
& \left.J_{y y \omega \omega}=\int_{A} y^{2} \omega^{2} d A, J_{x x y}=\int_{A} x^{2} y d A \quad J_{x y y \omega}=\int_{A} x y^{2} \omega d A, \quad \mathrm{~b}, \mathrm{c}, \mathrm{d}\right)
\end{aligned}
$$

where $W$ is the Wagner coefficient.

\section{ARCHES IN UNIFORM BENDING}

\subsection{Buckling Equation}

Fig. (3) shows a circular arch of radius $R$ subjected to two equal and opposite end moments. When a simply supported thin-walled arch is subjected to uniform bending $M_{X}=M$, the axial stress resultant is $F_{z}=0$. constant of the cross section.

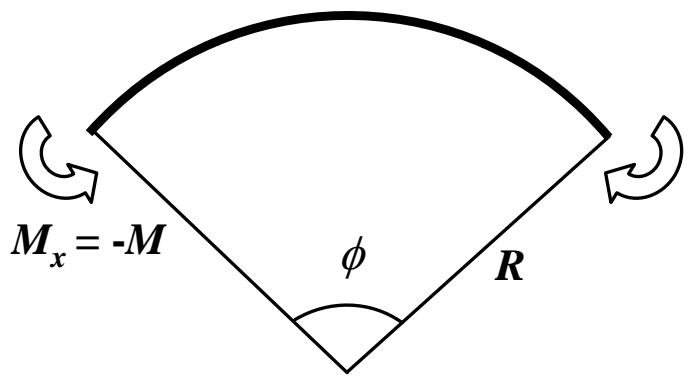

(b) Negative Bending

The prebuckling curvature due to uniform bending is constant along the arch axis, and can be obtained from Eq. (11) as

$\bar{v}_{o p}^{\prime \prime}=-\frac{M}{E I_{x}}$

from which the rotation $\bar{v}_{o p}^{\prime}$ along arch axis can be obtained as

$$
\bar{v}_{o p}^{\prime}=-\frac{M}{E I_{x}}\left(z-\frac{L}{2}\right)
$$

By substituting Eqs. (20) and (21) into the Eq. (18), the energy equation for the flexural-torsional buckling of arches subjected to the uniform bending is obtained. The buckling displacements for the laterally simply supported arch may take the form of

$$
\delta u_{o b}=a \sin \frac{\pi}{L} z, \delta \theta_{b}=b \sin \frac{\pi}{L} z
$$

where $a, b$ are the maximum values of $u_{o b}$ and $\theta_{b}$, respectively. Substituting Eqs. (20), (21), and (22) into Eq. (18) and recasting into matrix format produces

$$
\frac{L}{2}\left(\frac{\pi}{L}\right)^{2}\left\{\begin{array}{l}
a \\
b
\end{array}\right\}^{T}\left[\begin{array}{ll}
K_{11} & K_{12} \\
K_{21} & K_{22}
\end{array}\right]\left\{\begin{array}{l}
a \\
b
\end{array}\right\}=0
$$

where

$$
\begin{aligned}
& K_{11}=P_{y}+\frac{1}{R^{2}} r_{o}^{2} P_{\theta}+\frac{\chi_{1}}{R} M+\frac{E J_{y y \omega \omega}}{R^{4}}-\frac{M}{R} \frac{I_{y}}{I_{x}}+M \lambda^{2} \xi_{1} \\
& \mathrm{~K}_{12}=\mathrm{K}_{21}=-\frac{\mathrm{P}_{\mathrm{y}}}{\Psi \lambda}-\frac{1}{\mathrm{R}} \mathrm{r}_{\mathrm{o}}^{2} \mathrm{P}_{\theta}-\mathrm{M}\left(1+\chi_{1}\right)-\frac{1}{2 \mathrm{R}^{3}} \mathrm{EJ}_{\mathrm{yy} \omega \omega}+\frac{\mathrm{I}_{\mathrm{y}}}{\mathrm{I}_{\mathrm{x}}}\left(\mathrm{M}+\frac{\mathrm{M}}{2 \Psi^{2}}\right)+\frac{\mathrm{M}}{2 \Psi^{2}} \frac{\mathrm{GK}_{\mathrm{T}}}{\mathrm{EI}_{\mathrm{x}}}+\mathrm{M}_{2}+\frac{\mathrm{M}^{2}}{2 \mathrm{EI}_{\mathrm{x}}}\left[\xi_{1}\left(1+\frac{1}{\Psi^{2}}\right)+\frac{1}{\Psi \lambda}\right](24) \\
& \mathrm{K}_{22}=\frac{\mathrm{P}_{\mathrm{y}}}{\Psi^{2} \lambda^{2}}+\mathrm{r}_{\mathrm{o}}^{2} \mathrm{P}_{\theta}+\mathrm{M}\left(\chi_{2}+\frac{\chi_{3}}{\lambda^{2}}\right)+\frac{\mathrm{I}_{\mathrm{y}}}{\mathrm{I}_{\mathrm{x}}}\left(\frac{\mathrm{M}^{2}}{\mathrm{EI}_{\mathrm{x}} \lambda^{2}}-\frac{2 \mathrm{M}}{\Psi \lambda}\right)-\frac{\mathrm{I}_{\omega}}{\mathrm{I}_{\mathrm{x}}} \frac{2 \mathrm{M}}{\mathrm{R}}-\frac{\alpha_{3} \mathrm{M}}{\Psi^{2}}-\frac{\mathrm{GK}_{\mathrm{T}}}{\mathrm{EI}_{\mathrm{x}}} \frac{\mathrm{M}}{\Psi \lambda}-\frac{\mathrm{M}^{2}}{\mathrm{EI}_{\mathrm{x}} \lambda^{2}}\left(1+\xi_{3}\right)+\mathrm{M}_{4}
\end{aligned}
$$


in which

$$
\begin{aligned}
& \chi_{1}=-\frac{2 \alpha_{1}}{R^{2}}+\frac{\alpha_{2}}{R^{3}}+\frac{\alpha_{3}}{R}, \chi_{2}=\beta_{x}-\frac{2 \alpha_{1}}{R}+\frac{\alpha_{2}}{R^{2}}, \chi_{3}=\frac{1}{R}+\frac{\alpha_{3}}{R^{2}} \\
& \text { (25a, b, c) } \\
& \xi_{1}=-\frac{2 \alpha_{1}}{R}+\frac{\alpha_{2}}{R^{2}}+\alpha_{3} \\
& \xi_{2}=\alpha_{1}\left(\lambda^{2}+\frac{1}{R^{2}}\right)-\frac{\alpha_{2} \lambda^{2}}{R}+\frac{\alpha_{3}}{2 R}\left(1-\frac{1}{\Psi^{2}}\right) \\
& \xi_{3}=\frac{2 y_{o}}{R}-\alpha_{1}\left(\lambda^{2}+\frac{3}{R^{2}}\right)-\frac{\alpha_{2}}{R}\left(\frac{1}{R^{2}}-\lambda^{2}\right)+\frac{2}{R}\left(\alpha_{3}-\alpha_{4}\right), \\
& \xi_{4}=-\frac{2 \alpha_{1}}{R}+\alpha_{2} \lambda^{2}+\alpha_{3} \\
& \alpha_{1}=\frac{J_{x y \omega}}{I_{x}}, \alpha_{2}=\frac{J_{\omega \omega y}}{I_{x}}, \alpha_{3}=\frac{J_{x x y}}{I_{x}}, \alpha_{4}=\frac{J_{x}}{I_{x}}(27 \mathrm{a}, \mathrm{b}, \mathrm{c}, \mathrm{d}) \\
& J_{x y \omega}=\int_{A} x y \omega d A, J_{x}=\int_{A} y^{3} d A \\
& P_{y}=E I_{y} \lambda^{2} \\
& r_{o}^{2}=\frac{1}{A}\left(I_{x}+I_{y}+A y_{o}^{2}\right), \Psi=R \lambda \\
& P_{\theta}=\frac{1}{r_{o}^{2}}\left(E I_{\omega} \lambda^{2}+G K_{T}\right) \\
& \beta_{x}=\frac{J_{x}}{I_{x}}+\frac{J_{x x y}}{I_{x}}-2 y_{o}
\end{aligned}
$$

where $P_{y}$ is the minor axis flexural buckling load for a straight column; $P_{\theta}$ is the torsional buckling load for a straight column; $r_{o}$ denotes the radius of gyration with respect to the shear center; $\lambda=n \pi / L$; and $n$ is the number of buckled half waves around the arc length $L ; \beta_{x}$ denotes the monosymmetry parameter.

Eq. (23) has a non-trivial solution for $a$ and $b$ when

$K_{11} K_{22}-K_{12} K_{21}=0$

which leads to the quartic equation

$$
A_{1} M_{c r}^{4}+A_{2} M_{c r}^{3}+A_{3} M_{c r}^{2}+A_{4} M_{c r}+A_{5}=0
$$

where the coefficients $A_{1}, A_{2}, A_{3}, A_{4}$, and $A_{5}$ are given in Appendix.

When an infinite radius of curvature is used for Eq. (31), the monosymmetric arch degenerates to a straight beam of monosymmetric cross-section, and the buckling equation for monosymmetric straight beam is given by Eq. (32).

in which $M_{c r-M S B-p}$ is the critical buckling moment for monosymmetric straight beam considering the effects of prebuckling deformations. In Eq. (32), the terms $\left(\frac{\alpha_{3}}{2 E I_{x}}\right)^{2}, \alpha_{1} \lambda^{2}\left(2-\alpha_{1} \lambda^{2}\right)$, and $\alpha_{3} \lambda^{2}\left(\beta_{x}+\alpha_{2}\right)$ are very small and can be neglected without affecting the results so that Eq. (32) can be reduced as eq. (33).

Eq. (33) is in close agreement with Pi et al. [16]. When the effects of prebuckling deformations on straight beams are ignored, the classical buckling moment $\left(M_{c r-M S B}\right)$ for a monosymmetric straight beam can be obtained as

$-M_{c r-M S B}^{2}+P_{y} \beta_{x} M_{c r-M S B}+r_{o}^{2} P_{y} P_{\theta}=0$

\subsection{Effects of Prebuckling}

The comparative study on the prebuckling effects is performed by using the monosymmetric I-section shown in Fig. (4) with typical material properties (Young's Modulus; $E=63,000 \mathrm{MPa}$, Shear Modulus; $G=27,000 \mathrm{MPa}$; theses values are also used throughout this section) and the developed length of the arch is $L=1,000 \mathrm{~mm}$.

The closed form solution of Eq. (31) for the flexuraltorsional buckling of the laterally pinned circular arch with the section-B in Fig. (4) is compared with that of Pi et al. [16]. Fig. (5) shows the variation of the buckling moment ratio $M_{c r} / M_{c r-M S B}$ with the subtended angle, and the classical buckling moment $M_{c r-M S B}$ of a straight beam of monosymmetric section can be calculated from Eq. (34). When the prebuckling deformation is ignored, the solutions of Eq. (31) closely agree with that of Pi et al. [16]. When the prebuckling deformation is considered, the solutions of Eq. (31) generally close to that of quadratic equation by $\mathrm{Pi}$ et al. [16] in positive uniform bending, but disagrees with that of $\mathrm{Pi}$ et al. [8] for the arch with a large subtended angle in negative uniform bending. As shown in Fig. (5a), for straight beam with section-B in positive bending, the classical buckling moment $\left(M_{c r-M S B}\right)$ is $18.7 \mathrm{~N}-\mathrm{m}$, while the buckling moment considering the prebuckling $\left(M_{c r-M S B-p}\right)$ is $23.8 \mathrm{~N}-\mathrm{m}$, which is about $27 \%$ higher. In case of the arch (subtended angle is 180 degree) in Fig. (5b), the classical buckling moment is $152 \mathrm{~N}-\mathrm{m}$, while the buckling moment considering the prebuckling $\left(M_{c r}\right)$ is $239 \mathrm{~N}-\mathrm{m}$, which is about $57.2 \%$ higher.

In deriving the cubic buckling equation by Pi et al. [16], prebuckling terms including $\left(I_{y} / I_{x}\right)^{2}$ and $\left(I_{y} / I_{x}\right)^{3}$ in the coefficient of the cubic term were neglected. Also, using the assumption that the cubic term can be ignored because the co-

$$
\begin{aligned}
& \left(\frac{\alpha_{3}}{2 \mathrm{EI}_{\mathrm{x}}}\right)^{2} \mathrm{M}_{\mathrm{Cr}-\mathrm{MSB}-\mathrm{p}}^{4}+\left[\frac{\mathrm{I}_{\mathrm{y}}}{\mathrm{I}_{\mathrm{x}}}-1+\alpha_{1} \lambda^{2}\left(2-\alpha_{1} \lambda^{2}\right)+\alpha_{3} \lambda^{2}\left(\beta_{\mathrm{x}}+\alpha_{2}\right)\right] \mathrm{M}_{\mathrm{cr}-\mathrm{MSB}-\mathrm{p}}^{2}+\left[\mathrm{P}_{\mathrm{y}}\left(\beta_{\mathrm{x}}+\alpha_{2} \lambda^{2}+\alpha_{3}\right)+\mathrm{r}_{\mathrm{o}}^{2} \mathrm{P}_{\theta} \alpha_{3} \lambda^{2}\right] \mathrm{M}_{\mathrm{cr}-\mathrm{MSB}-\mathrm{p}}+\mathrm{r}_{\mathrm{o}}^{2} \mathrm{P}_{\mathrm{y}} \mathrm{P}_{\theta}=0 \\
& \left(\frac{I_{y}}{I_{x}}-1\right) M_{c r-M S B-p}^{2}+\left[P_{y}\left(\beta_{x}+\alpha_{2} \lambda^{2}+\alpha_{3}\right)+r_{o}^{2} P_{\theta} \alpha_{3} \lambda^{2}\right] M_{c r-M S B-p}+r_{o}^{2} P_{y} P_{\theta}=0
\end{aligned}
$$




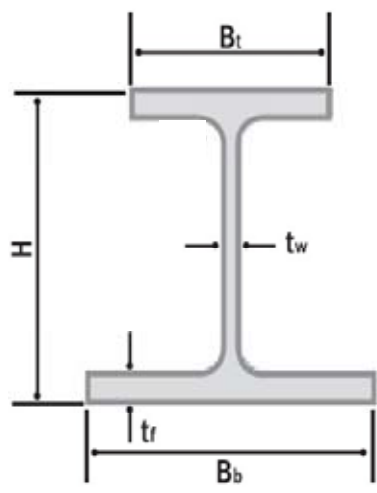

(a) Cross section

Fig. (4). Cross section and dimensions.

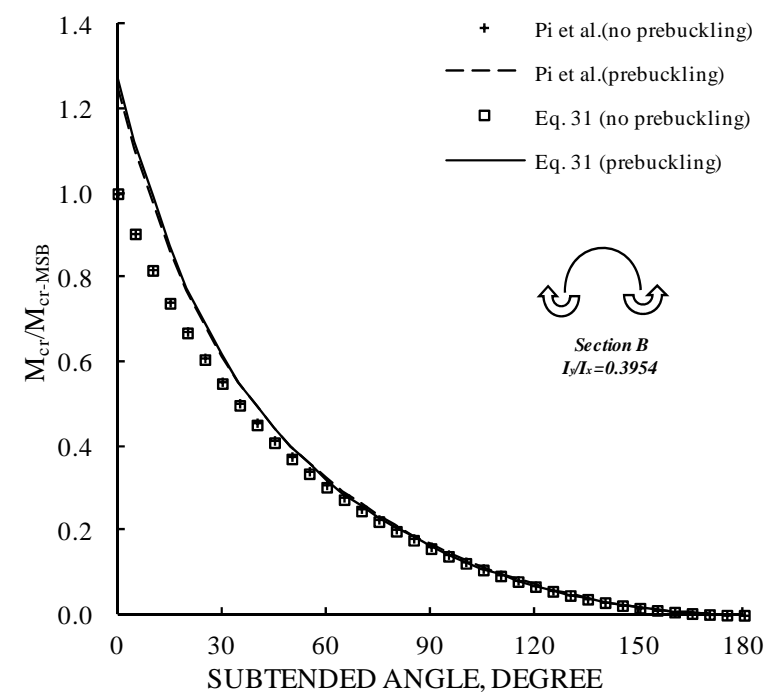

(a) Positive Uniform Bending

Fig. (5). Buckling of monosymmetric arch in uniform bending.

efficient of the cubic term is very small, the cubic buckling equation was reduced to the quadratic equation in the study of Pi et al. [16]. The solutions of the cubic and quadratic buckling equation by Pi et al. [16] are compared with those of Eq. (31). Fig. (6) shows the variation of the buckling moment ratio $M_{c r} / M_{c r-M S B}$ with the subtended angle. When the arch is subjected to the positive uniform bending moment, the solutions of the cubic and quadratic buckling equation by Pi et al. [16] closely agree with those of Eq. (31) without reference to the ratio of $I_{y} / I_{x}$ of the minor axis flexural stiffness to the major axis flexural stiffness. However, in case of the negative uniform bending, the solutions of $\mathrm{Pi}$ et $a l$. [16] disagree with Eq. (31) as the subtended angle and the ratio of $I_{y} / I_{x}$ increase. The buckling moment predicted by the cubic equation by $\mathrm{Pi}$ et al. [16] is 6.13\% higher than the Eq. (31) for Section-C with $\phi=180^{\circ}$, and the buckling moment of the quadratic equation by $\mathrm{Pi}$ et al. [16] is $13.9 \%$ higher than the Eq. (31). Therefore, as the subtended angle

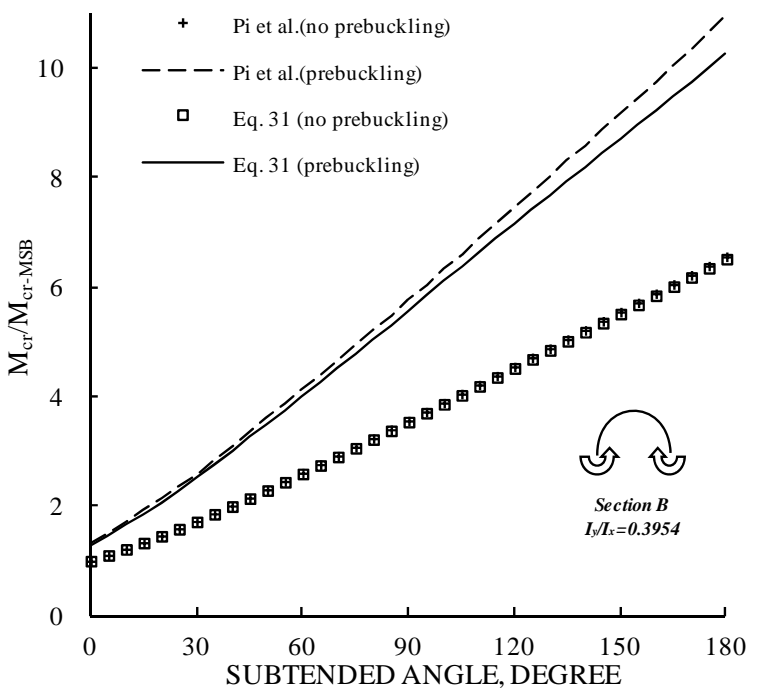

(b) Negative Uniform Bending

increases, the quadratic buckling equation by $\mathrm{Pi}$ et al. [16] must be used with caution when the ratio of $I_{y} / I_{x}$ is not small. Also it is found that the buckling equation of Pi et al. [16] estimate the flexural-torsional buckling strength too highly, and this is because that the terms referring to the prebuckling deformation with curvature effects such as $\vec{v}_{o}^{\prime} u_{o}^{\prime} / R$ in Eq. (5) are not considered in the straindisplacement relations of Pi et al. [16].

In order to investigate the influence of the higher order prebuckling terms with curvature effects on the buckling resistance of arches, the solutions of Eq. (31) are compared with the approximate solutions of Eq. (31). The approximate solution can be obtained by neglecting the higher order prebuckling terms with curvature such as $\vec{v}_{o}^{\prime} \theta u_{o}^{\prime} / R, \vec{v}_{o}^{\prime \prime} \theta^{2} / R$ from the nonlinear strain-displacement relationship of Eq. (5). The variations of the buckling moment ratio of the monosymmetric arch in uniform bending moment are plotted 


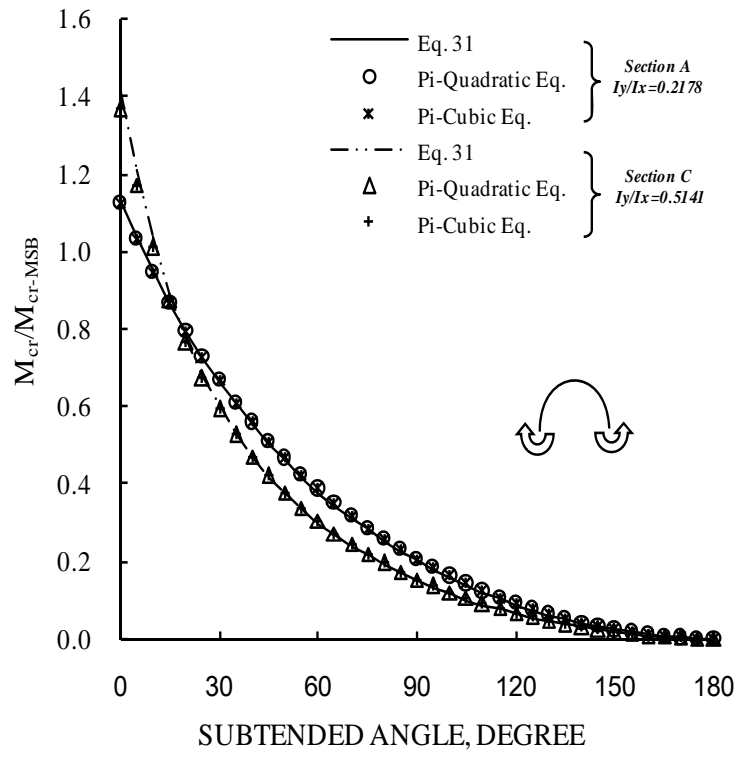

(a) Positive Uniform Bending

Fig. (6). Comparison of present results with those of Pi et al. [8].

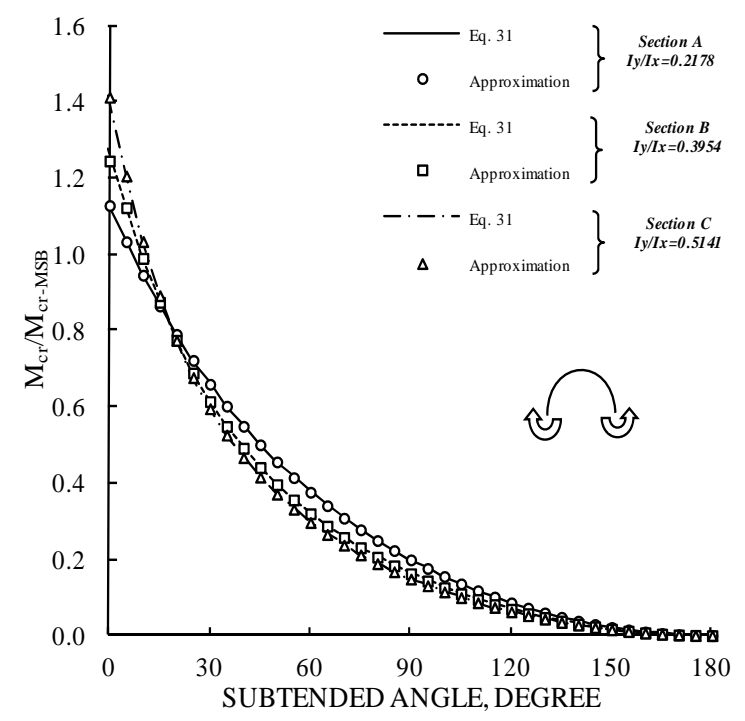

(a) Positive Uniform Bending

Fig. (7). Effects of the higher order prebuckling terms with curvature.

in Fig. (7) with varying the ratio of $I_{y} / I_{x}$. As shown in Fig. (7), the approximate solutions of Eq. (31) agree well with the accurate Eq. (31) except for a large subtended angle or small radius of curvature. Therefore, the approximate solutions of Eq. (31) can be used to predict the flexural-torsional buckling moment of the arch without a significant loss of accuracy.

\section{CONCLUSIONS}

In this paper, the elastic flexural-torsional buckling of monosymmetric arches subjected to the uniform bending has been investigated by considering the effects of prebuckling deformations. After using the nonlinear strain-displacement

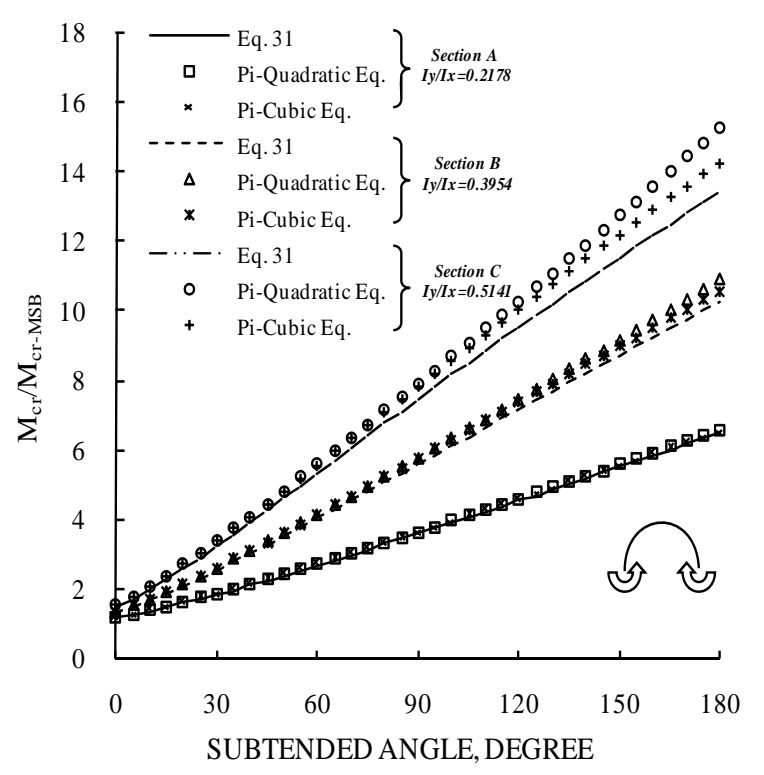

(b) Negative Uniform Bending

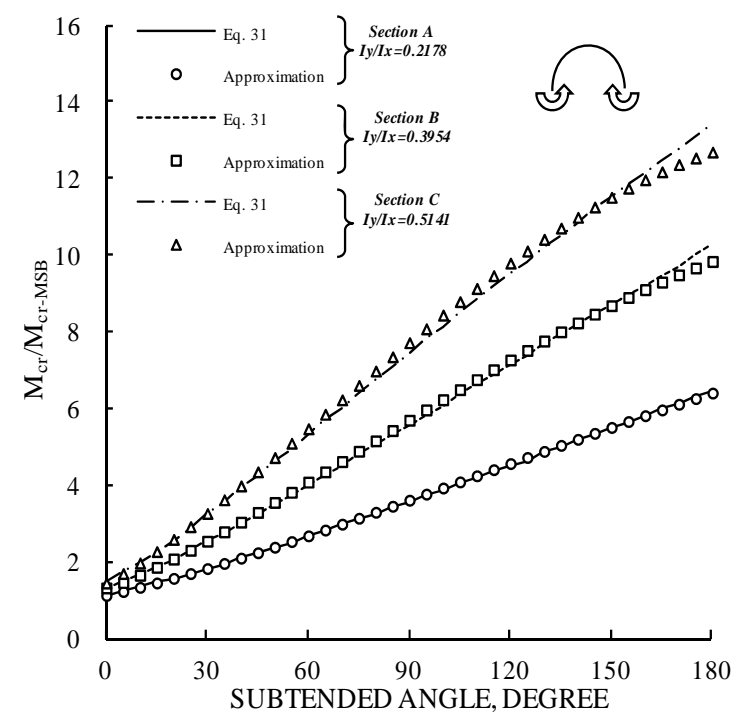

(b) Negative Uniform Bending

relations considering initial curvature effects and higher order prebuckling deformation terms with curvature in deriving process, closed form solution for the buckling moment has been obtained based on the energy method.

When the prebuckling deformation is ignored, the quartic equation of Eq. (31) closely agrees with that of Pi et al. [16]. When the prebuckling deformation is considered, the proposed quartic equation generally close to that of buckling equation by Pi et al. [16] in positive uniform bending, but disagrees with that of Pi et al. [16] for the arch with a large subtended angle in negative uniform bending. As the subtended angle increases, the quadratic buckling equation proposed by Pi et al. [16] must be used with caution when the 
ratio of $I_{y} / I_{x}$ is not small. Since the terms referring to the prebuckling deformation with curvature effects such as $\vec{v}_{o}^{\prime} u_{o}^{\prime} / R$ are not considered in the strain-displacement relations of Pi et al. [16], the buckling equation of Pi et al. [16] can slightly overestimate the flexural-torsional buckling strength of monosymmteric arches.

By neglecting the higher order prebuckling terms with curvature from the nonlinear strain-displacement relationship, the accurate quartic equation of Eq. (31) has been simplified as the approximated form. It is found that the higher order prebuckling terms with curvature has no effects on the buckling strength of monosymmteric arches. Therefore, the APPENDIX

The quartic equation is given by

$$
A_{1} M_{c r}^{4}+A_{2} M_{c r}^{3}+A_{3} M_{c r}^{2}+A_{4} M_{c r}+A_{5}=0
$$

where the coefficients $A_{1}, A_{2}, A_{3}, A_{4}$, and $A_{5}$ are given by

$$
\begin{aligned}
& A_{1}=\left\{\frac{1}{2 E I_{x}}\left[\xi_{1}\left(1+\frac{1}{\Psi^{2}}\right)+\frac{1}{\Psi \lambda}\right]\right\}^{2} \\
& A_{2}=\frac{I_{y}}{I_{x}} \frac{1}{E I_{x}}\left[\frac{1}{\Psi}\left(\frac{2 y_{o}}{\Psi}-\frac{1}{\lambda}-\frac{1}{2 \Psi^{2} \lambda}\right)+\kappa_{1}\right] \\
& +\frac{1}{E I_{X}}\left\{\frac{1}{\Psi}\left(\frac{1}{\lambda}+\frac{1}{\Psi}\right)-\frac{1}{2 \Psi^{2}} \frac{G K_{T}}{E I_{X}}\left[\xi_{1}\left(1+\frac{1}{\Psi^{2}}\right)+\frac{1}{\Psi \lambda}\right]+\frac{2 \alpha_{4}}{R} \xi_{1}+\kappa_{2}\right\} \\
& A_{3}=-\left(1+\chi_{1}\right)^{2}+\frac{1}{R} \chi_{1} \chi_{2}+\frac{1}{\Psi \lambda} \chi_{1} \chi_{3}+\frac{r_{o}^{2} P_{\theta}}{E I_{x}}\left[\frac{1}{\Psi^{2}}\left(\frac{I_{y}}{I_{x}}-\frac{2 y_{o}}{R}\right)+\kappa_{3}\right] \\
& +\frac{G K_{T}}{E I_{X}}\left[\frac{1}{\Psi^{2}}\left(1-\frac{1}{4 \Psi^{2}} \frac{G K_{T}}{E I_{X}}\right)+\kappa_{4}\right]-\frac{I_{\omega}}{I_{X}}\left[\frac{2}{R}\left(\lambda^{2} \xi_{1}+\frac{\chi_{1}}{R}\right)\right] \\
& +\frac{I_{y}}{I_{x}}\left[1+\frac{I_{y}}{I_{x}} \frac{1}{\Psi^{2}}\left(1-\frac{1}{4 \Psi^{2}}\right)+\frac{2}{R^{2}} \frac{I_{\omega}}{I_{x}}-\frac{1}{2 \Psi^{4}} \frac{G K_{T}}{E I_{x}}-\frac{1}{R}\left(\beta_{x}+2 y_{o}\right)+\frac{1}{\Psi^{2}}\left(1+\frac{1}{R}\right)+\kappa_{5}\right] \\
& -\frac{J_{y y \omega \omega}}{I_{x}}\left[\frac{1}{R^{2} \Psi^{2}}\left(\frac{1}{2}+\frac{2 y_{o}}{R}\right)+\kappa_{6}\right]+\beta_{x} \lambda^{2} \xi_{1}+\kappa_{7} \\
& A_{4}=\chi_{1}\left(\frac{r_{o}^{2} P_{\theta}}{R}+\frac{P_{y}}{\Psi^{3} \lambda}\right)-2\left(1+\chi_{1}\right)\left(\frac{r_{o}^{2} P_{\theta}}{R}+\frac{P_{y}}{\Psi \lambda}\right)+\chi_{2}\left(P_{y}+\frac{r_{o}^{2} P_{\theta}}{R^{2}}\right)+\chi_{3}\left(\frac{P_{y}}{\lambda^{2}}+\frac{r_{o}^{2} P_{\theta}}{\Psi^{2}}\right) \\
& -\frac{I_{\omega}}{I_{x}}\left(\frac{2 E J_{y y \omega \omega}}{R^{5}}+\frac{2 P_{y}}{R}\right)+\frac{I_{y}}{I_{x}}\left[\frac{E J_{y y \omega \omega}}{R^{3}}\left(1-\frac{3}{2 \Psi^{2}}\right)+\frac{r_{o}^{2} P_{\theta}}{R}\left(1-\frac{r_{o}^{2} P_{\theta}}{\Psi^{2}}\right)\right] \\
& +r_{o}^{2} P_{\theta}\left[\alpha_{3}\left(\lambda^{2}+\frac{2}{R^{2}}-\frac{2}{R^{2} \Psi^{2}}\right)-\frac{2}{R^{3}} \frac{I_{\omega}}{I_{x}}\right]+\frac{E J_{y y \omega \omega}}{R^{3}}\left[\frac{\chi_{2}}{R^{4}}-1-\frac{1}{\Psi^{2}}\left(\frac{G K_{T}}{2 E I_{x}}+1\right)+\kappa_{8}\right] \\
& +P_{y}\left[\frac{G K_{T}}{E I_{x}} \frac{1}{\Psi \lambda}\left(\frac{1}{\Psi^{2}}-1\right)+\kappa_{9}\right] \\
& A_{5}=r_{o}^{2} P_{y} P_{\theta}\left(1-\frac{1}{\Psi^{2}}\right)^{2}+\frac{E J_{y y \omega \omega}}{R^{4}}\left[\frac{P_{y}}{\lambda^{2}}\left(\frac{1}{\Psi^{2}}-1\right)-\frac{E J_{y y \omega \omega}}{4 R^{2}}\right]
\end{aligned}
$$


in which

$$
\begin{aligned}
& \kappa_{1}=\frac{\alpha_{1}}{R}\left[\frac{1}{\Psi^{2}}\left(\frac{1}{\Psi^{2}}-2\right)-1\right]+\frac{\alpha_{2}}{R^{2}}\left[1-\frac{1}{2 \Psi^{2}}\left(3+\frac{1}{\Psi^{2}}\right)\right]+\frac{\alpha_{3}}{\Psi^{2}}\left(1-\frac{1}{2 \Psi^{2}}\right)-\frac{\alpha_{4}}{\Psi^{2}} \\
& \kappa_{2}=\frac{\alpha_{1}}{R}\left[\frac{4 y_{o}}{R}-\frac{1}{\Psi^{2}}\left(3+\frac{2}{R}\right)-1+\frac{2 \alpha_{1}}{R^{2}}\left(1+\frac{1}{\Psi^{2}}+2 \Psi^{2}\right)\right]+\frac{\alpha_{2}}{R^{2}}\left[1+\frac{1}{\Psi^{2}}\left(1+\frac{1}{R}\right)-\frac{2 y_{o}}{R}+\frac{3 \alpha_{2}}{R^{3}}\right] \\
& +\frac{\alpha_{3}}{R}\left[\frac{1}{\Psi}\left(\frac{1}{2 \lambda}+\frac{1}{\Psi}+\frac{1}{2 \Psi^{2} \lambda}\right)-2 y_{o}+\frac{\alpha_{3}}{2}\left(\frac{1}{\Psi^{4}}-3\right)\right]-\frac{\alpha_{1} \alpha_{2}}{R^{4}}\left(7+\frac{1}{\Psi^{2}}\right) \\
& +\frac{\alpha_{1} \alpha_{3}}{R^{2}}\left[2-\frac{1}{\Psi^{2}}\left(1+\frac{1}{\Psi^{2}}\right)\right]+\frac{\alpha_{2} \alpha_{3}}{2 R^{3}}\left(\frac{1}{\Psi^{4}}+3\right) \\
& \kappa_{3}=\frac{\alpha_{1}}{R^{2}}\left(\frac{1}{\Psi^{2}}-3\right)+\frac{2 \alpha_{2}}{R^{3}}\left(1+\frac{1}{\Psi^{2}}\right)+\frac{\alpha_{3}}{R}\left(1-\frac{1}{\Psi^{2}}\right)+\frac{2 \alpha_{4}}{R \Psi^{2}} \\
& \kappa_{4}=\frac{\alpha_{1}}{R^{2}}\left(1-\frac{1}{\Psi^{2}}\right)-\frac{\alpha_{3}}{R}\left[1+\frac{1}{2 \Psi^{2}}\left(1-\frac{1}{\Psi^{2}}\right)\right] \\
& \kappa_{5}=\frac{\alpha_{1}}{R^{2}}\left(2-\Psi^{2}-\frac{1}{\Psi^{2}}\right)+\frac{2 \alpha_{2}}{R^{3}}+\frac{\alpha_{3}}{R}\left[\frac{1}{2 \Psi^{2}}\left(\frac{1}{\Psi^{2}}-1\right)-3\right]+\frac{2 \alpha_{4}}{R} \\
& \kappa_{6}=\frac{2 \alpha_{1}}{R^{4}}\left(1-\frac{1}{\Psi^{2}}\right)-\frac{3 \alpha_{2}}{2 R^{5}}\left(\frac{1}{\Psi^{2}}+1\right)-\frac{\alpha_{3}}{2 R^{3}}\left(1-\frac{3}{\Psi^{2}}\right)-\frac{2 \alpha_{4}}{R^{3} \Psi^{2}} \\
& \kappa_{7}=\alpha_{1}\left[2 \lambda^{2}-\alpha_{1}\left(\lambda^{4}+\frac{1}{R^{4}}-\frac{2 \lambda^{2}}{R^{2}}\right)\right]+\frac{\alpha_{2}}{R}\left(\frac{1}{R^{2}}-2 \lambda^{2}\right)+\frac{\alpha_{3}}{R}\left(2-\frac{1}{\Psi^{2}}\right) \\
& +\left(\frac{\alpha_{3}}{R}\right)^{2}\left[\frac{7}{4}+\Psi^{2}-\frac{1}{2 \Psi^{2}}\left(5+\frac{1}{2 R^{3} \Psi^{2}}\right)\right]+\frac{\alpha_{1} \alpha_{3}}{R}\left[\frac{1}{R^{2}}\left(\frac{5}{\Psi^{2}}-4\right)-5 \lambda^{2}\right] \\
& +\alpha_{2} \alpha_{3}\left[\frac{1}{R^{4}}\left(1+2 \Psi^{2}-\frac{2}{\Psi^{2}}\right)+\lambda^{4}\right] \\
& \kappa_{8}=\alpha_{1}\left(\lambda^{2}+\frac{1}{R^{2}}\right)-\frac{\alpha_{2}}{R^{3}}+\frac{\alpha_{3}}{2 R}\left(1-\frac{1}{\Psi^{2}}\right) \\
& \kappa_{9}=\alpha_{2}\left(\lambda^{2}-\frac{2}{R^{2}}\right)+\alpha_{3}\left[1-\frac{1}{\Psi^{2}}\left(1+\frac{1}{\Psi^{2}}\right)\right]
\end{aligned}
$$

\section{REFERENCES}

[1] N.S. Trahair, and S.T. Woolcock, "Effect of major axis curvature on I-beam stability” J. Eng. Mech. Div., ASCE, vol. 99, no. 1, pp. 85-98, 1973.

[2] T.M. Roberts, and Z.G. Azzizian, "Influence of pre-buckling displacements on the elastic critical loads of thin walled bars of open cross section” Int. J. Mech. Sci., vol. 25, no. 2, pp. 93-104, 1983.

[3] Y-L. Pi, and N.S. Trahair, "Prebuckling deflections and lateral buckling. I: theory” J. Struct. Eng., ASCE, vol. 118, no. 11, pp. 2949-2966, 1992.

[4] Y-L. Pi, and N.S. Trahair, "Prebuckling deflections and lateral buckling. II: application” J. Struct. Eng., ASCE, vol. 118, no. 11, pp. 2967-2985, 1992.

[5] V.Z. Vlasov, Thin-walled elastic beams. Israel Jerusalem: Program for Scientific Translation, 1961.

[6] C.H. Yoo, "Flexural-torsional stability of curved beams." J. Eng. Mech. Div., ASCE, vol. 108, no. 6, pp. 1351-1369, 1982.

[7] J.P. Papangelis, and N.S. Trahair, "Flexural-torsional buckling of arche” J. Struct. Eng., ASCE, vol. 113, no. 4, pp. 889-906, 1987.

[8] Y.J. Kang, and C. H. Yoo, "Thin-walled curved beams I: Formulation of nonlinear equations” J. Eng. Mech., ASCE, vol. 120, no.10, pp. 2072-2101, 1994.
[9] Pi, Y-L. and Bradford, M.A, "Elastic flexural-torsional buckling of continuously restrained arches.” Int. J. Solids Structures, vol. 39, pp. 2299-2322, 2002.

[10] Y-L. Pi, and M.A. Bradford, "Elastic flexural-torsional buckling of fixed arches” Q. J. Mech. Appl. Math., Oxford University Press, vol. 57, no. 4, pp. 551-569, 2004

[12] M.A. Bradford, and Y-L. Pi, "Elastic flexural-torsional instability of structural arches under hydrostatic pressure” Int. J. Mech. Sci., vol. 50, pp. 143-151, 2008.

[11] N-H. Lim, and Y.J. Kang, "Out of plane stability of circular arches” Int. J. Mech. Sci., vol. 46, pp. 1115-1137, 2004.

[13] Y. Yang, C. Chen, and M. Fang, "Lateral-torsional buckling strength and design of steel arches.” Adv. Mat. Res., TTP, 250-253, pp. 2645-2649, 2011.

[14] P. Vacharajittiphan, and N.S. Trahair, "Flexural-torsional buckling of curved members” J. Struct. Eng. Div., ASCE, vol. 101, no. 6, pp. 1223-1238, 1975.

[15] C.H. Yoo, and P.A. Pfeiffer, "Buckling of curved beams with inplane deformation.” J. Struct. Eng. Div., ASCE, vol. 110, no. 2, pp. 291-300, 1984.

[16] Y-L. Pi, J.P. Papangelis, and N.S. Trahair, "Prebuckling deformations and flexural-torsional buckling of arches" J. Struct. Eng., ASCE, vol. 121, no. 9, pp. 1313-1322, 1995. 
[17] Y-L. Pi, and M.A. Bradford, "Effects of prebuckling deformations on the elastic flexural-torsional buckling of laterally fixed arches" Int. J. Mech. Sci., vol. 46, pp. 321-342, 2004.

[18] Y-L. Pi, and M.A. Bradford, "Effects of prebuckling analyses on determining buckling loads of pin-ended circular arches" Mech. Res. Commun., vol. 37, pp. 545-553, 2010.
[19] T. Usami and S.Y. Koh, "Large displacement theory of thin-walled curved members and its application to lateral-torsional buckling analysis of circular arches" J. Solids Struct., vol. 16, pp. 71-95, 1980.

[20] J. Y. Oden, Mechanics of elastic structures. McGrow-Hill Book Co., Inc: USA, 1967.

Received: June 05, 2012

(C) Ryu et al.; Licensee Bentham Open.

This is an open access article licensed under the terms of the Creative Commons Attribution Non-Commercial License (http://creativecommons.org/licenses/by-nc/3.0/) which permits unrestricted, non-commercial use, distribution and reproduction in any medium, provided the work is properly cited. 